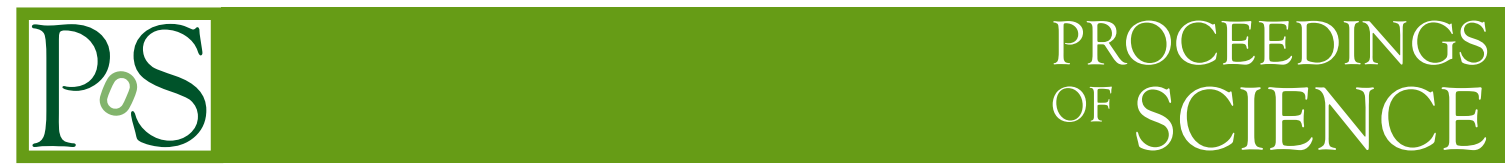

\title{
CMS results on Quarkonium production
}

\section{Jianguo Bian*}

Institute of High Energy Physics, Beijing, China

E-mail: bianjg@mail.ihep.ac.cn

This talk first presents the differential cross section measurements of prompt quarkonium states $J / \psi, \psi(2 S)$ and $\Upsilon(n S)$ in pp collisions at a center mass energy of $7 \mathrm{TeV}$, as a function of transverse momentum and in several rapidity, on the basis of the 2010 data collected by CMS; then reports the ratio of $\chi_{c 1}$ and $\chi_{c 2}$ cross sections as a function of transverse momentum, the ratio of $\mathrm{X}(3872)$ and $\psi(2 S)$ cross sections and the fraction of non-prompt $\mathrm{X}(3872)$ as a function of transverse momentum on the basis of the 2011 data; finally presents a structure $\mathrm{Y}(4140)$ in $J / \psi \phi$ mass in $B^{+} \rightarrow J / \psi \phi K^{+}$decay in 2011 data.

LHC on the March - IHEP-LHC,

20-22 November 2012

Institute for High Energy Physics, Protvino,Moscow region, Russia

\footnotetext{
*Speaker.
} 


\section{Introduction}

Observation of quarkonium states challenges QCD calculations. The cross sections of the quarkonium states $J / \psi, \psi(2 S)$ and $\Upsilon(n s)(n=1,2,3)$ can test the prediction of NLO QCD and NRQCD calculation. The measurements of $\mathrm{P}$ states $\chi_{c J}$ provide effective tests of heavy quarkonium production mechanisms based on NRQCD, in which nonperturbative effects can be simplified safely. Exotic states $(\mathrm{X}, \mathrm{Y}, \mathrm{Z})$ poses a serious challenge to the conventional quark model. X(3872) differential cross section measurement tests NRQCD prediction in a new energy range and a large pT reach. One need to understand the true nature of the exotic states such as hybrid, tetraquark state or molecular state.

The CMS experiment provides one more opportunity to observe quarkonia in proton-proton collisions thanks to its good muon detector and electromagnetic calorimeter. Two data sets of $40 \mathrm{pb}^{-1}$ and $5 \mathrm{fb}^{-1}$ for pp collisions at $\sqrt{s}=7 \mathrm{TeV}$ was recorded by CMS in 2010 and 2011 respectively. During this period a large sample of quarkonia was collected using inclusive trigger path for dimuon or multiple-muon events. The results presented in this paper are obtained by analyzing these data sets.

\section{Prompt $J / \psi$ and $\psi(2 S)$ cross sections}

On the basis of a dimuon event sample of $37 \mathrm{pb}^{-1}$ integrated luminosity, CMS measured the the $J / \psi$ and $\psi(2 S)$ cross sections ${ }^{[1]}$ as a function of transverse momentum and in several rapidity ranges. To separate the prompt components, where $c \bar{c}$ pair is produced promptly, from the non-prompt components, originating from B-meson decays, a unbinned log likelihood fit of 2 dimension is performed to the dimuon mass distribution and the proper decay length distribution. The proper decay length is defined as the transverse distance between the dimuon vertex and the primary vertex, corrected by the transverse Lorentz-boost of the dimuon. The measured prompt $J / \psi$ and $\psi(2 S)$ cross sections, including feed down effect, are shown in Figure 1 as a function of pT and in the various rapidity ranges. The measurements are in good agreement with NLO NRQCD calculation. $J / \psi^{[2]}$ theory includes color octet and feed down effect. $\psi(2 S)^{[2]}$ theory includes only color octet because of absence of feed down from heavier charmonium.

Ratio of $\psi(2 S)$ to $J / \psi$ as a function of pT is shown in Figure 2. No significant variation is over rapidity. It also includes the comparison with the NRQCD prediction.

\section{3. $\Upsilon(n S)$ differential cross sections}

Based on $36 \mathrm{pb}^{-1} 2010$ data, the $\Upsilon(n S)$ cross section ${ }^{[3]}$ are measured using two approaches The first is that a cross section in kinematic the region $0<\mathrm{pT}<50 \mathrm{GeV},|\mathrm{y}|<2.4$ is corrected for acceptance and efficiency as shown in Figure 3. Unpolarization is assumpted for acceptance correction and feed down from higher states are not corrected for.

The second approach is the fiducial cross section as a function of pT and y in Figure ฤ. A fiducial cross section is corrected for efficienies but no acceptance, defined within a restricted muon kinematic range. The advantage of a fiducial cross section is that no polarization uncertainties are involved. 

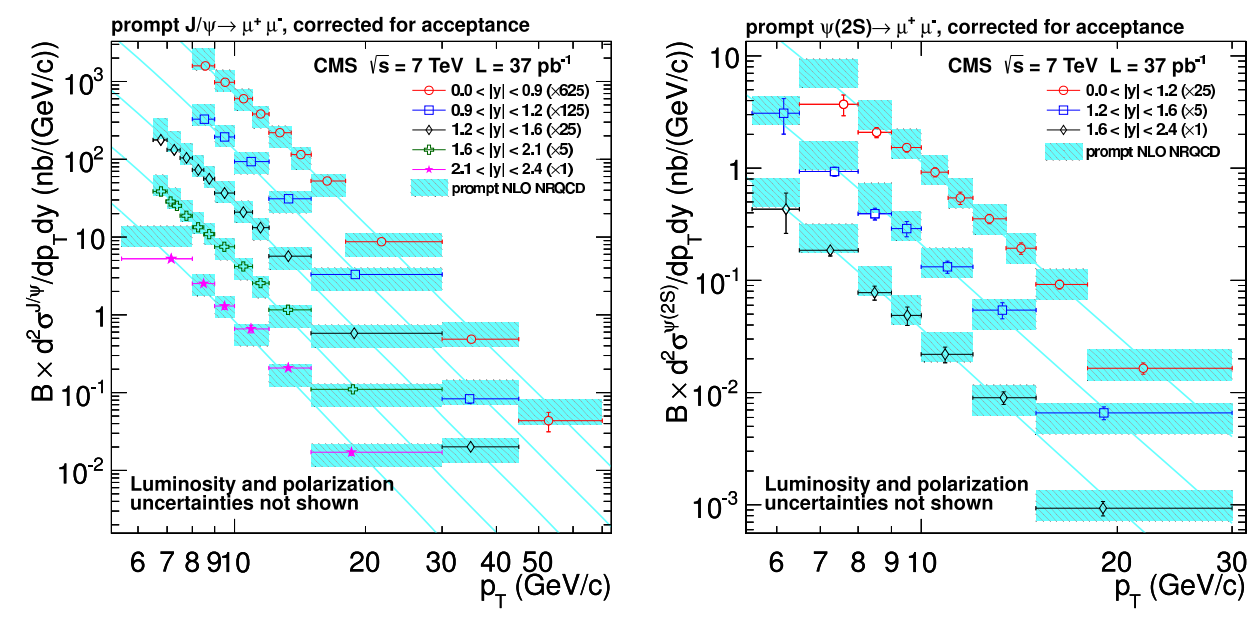

Figure 1: Measured differential cross section for prompt $J / \psi$ (left) and $\psi(2 S)$ (right) production as a function of pT for the different rapidity bins. The yellow and blue coloured histograms indicate the theoretical predictions from NRQCD calculations.

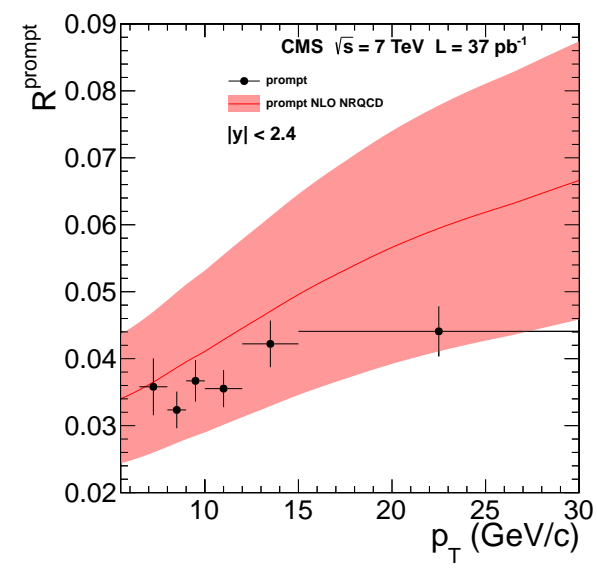

Figure 2: Measured value of the $\psi(2 S)$ to $J / \psi$ differential cross-section ratio, for prompt production, averaged over rapidity and plotted as a function of pT. It also includes the comparison with the NRQCD prediction. The shaded bands show the uncertainties on the theoretical predictions. The error bars give the total uncertainties on the measurements; polarization uncertainties are not included.

\section{Ratio of $\chi_{c 1}$ and $\chi_{c 2}$ cross sections}

On the basis of a dimuon event sample of $4.6 \mathrm{fb}^{-1}$ integrated luminosity recorded in 2011, the ratio of the $\mathrm{p}$ wave states $\chi_{c 1}$ and $\chi_{c 2}$ cross sections $^{[4]}$ as a function of transverse momentum are measured through the radiative decay $J / \psi+\gamma$ with $|\mathrm{y}(J / \psi)|<1.0$ and $\mathrm{pT}(\gamma)>0.5 \mathrm{GeV}$. Photons are reconstructed using the photon conversion reconstruction technique. High photon conversion rate, excellent photon momentum resolution and $\pi^{0}$ mass rejection bring excellent mass resolution of $10 \mathrm{MeV}$ to reslove the two states, see Figure 5. The NRQCD prediction is compatible with our data within the experimental and theoretical uncertainties. 

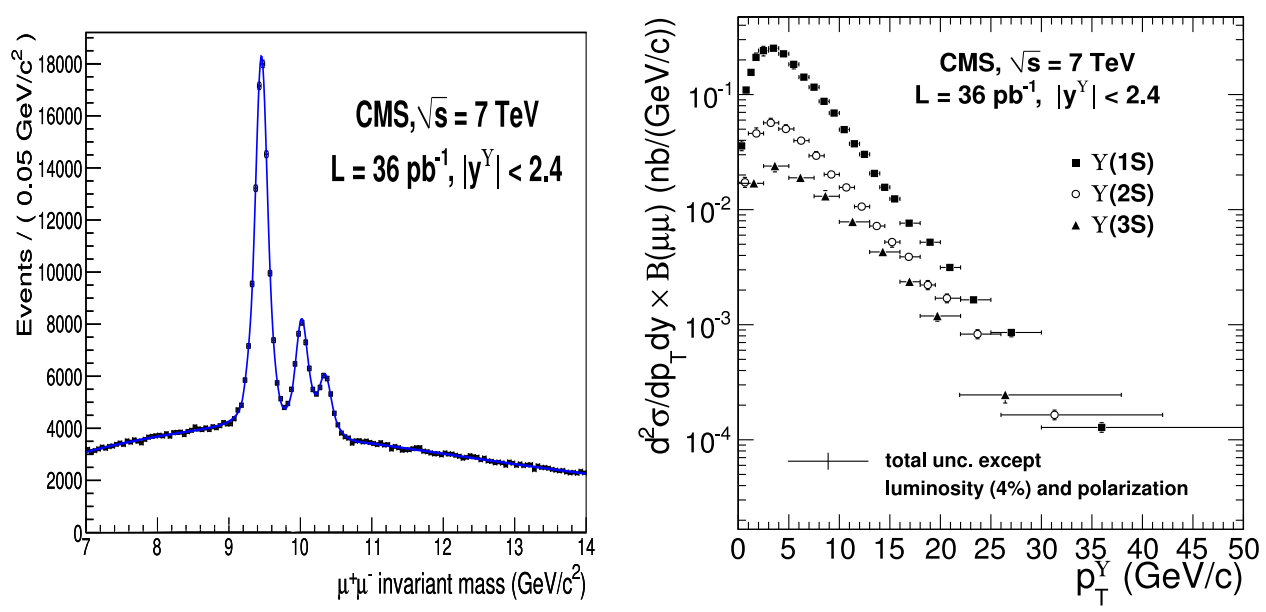

Figure 3: (Left) The dimuon invariant mass distribution in the vicinity of the $\Upsilon(n S)$ resonances for $-2.4<\mathrm{y}<2.4$. (Right) Differential cross sections of the $\Upsilon(n S)$ as a function of $\mathrm{pT}$ in the rapidity range $-2.4<\mathrm{y}<2.4$. The abscissa of the data points is the average $\mathrm{pT}$ in the interval.

\section{Ratio of $\mathbf{X}(3872)$ and $\psi(2 S)$ cross sections}

X(3872) was discovered by Bell in 2003 and confirmed by BaBar, CDF and D0. It was interpreted as $c \bar{c}$ state, $D^{*} \bar{D}^{0}$ molecule or tetra-quark state. On the basis of a dimuon event sample of $4.8 \mathrm{fb}^{-1}$ integrated luminosity, both $\mathrm{X}(3872)$ and $\psi(2 S)$, including prompt and non-prompt components, the latter is from B meson decays, were measured ${ }^{[5]}$ in $10<\mathrm{pT}(\mathrm{X})<50 \mathrm{GeV}$ and $|\mathrm{y}(\mathrm{X})|<1.2$. The ratio of the $\mathrm{X}(3872)$ to $\psi(2 S)$ is

$$
R=\frac{\left(p p \rightarrow \mathrm{X}(3872) B R\left(\mathrm{X}(3872) \rightarrow J / \psi \pi^{+} \pi^{-}\right)\right.}{(p p \rightarrow \psi(2 S)) B R\left(\psi(2 S) \rightarrow J / \psi \pi^{+} \pi^{-}\right)}=0.0656 \pm 0.0029 \pm 0.0065 .
$$

MC sample is based on pythia 6 with $\chi_{c 1}\left(J^{p c}=1^{++}\right)$mass set to $3.872 \mathrm{GeV}$ and unploarization. To estimate the non-prompt $\mathrm{X}(3872)$ fraction, which is the ratio of the signal in the B sample and the signal in the whole sample, pseudo-proper decay length is required to be $\ell_{x y}>100 \mu \mathrm{m}$ to enrich B hadron sample. The fraction is $0.263 \pm 0.023 \pm 0.016$, which has been corrected the efficiencies of the decay length selection criteria and not corrected for acceptance, see Figure 6 .

\section{Structures in the $J / \psi \phi$ spectrum}

The evidence of Y(4140) was reported by CDF in 2009 in $J / \psi \phi$ mass in $B^{+} \rightarrow J / \psi \phi K^{+}$ decay. Y(4140) is a candidate of an exotic state. It was not confirmed by Bell in 2010 and LHCb in 2011, while another structure Y(4350) was reported by Bell in 2010 in the same decay channel. CMS observed the two structure ${ }^{[6]}$ on the basis of a dimuon event sample of $5.2 \mathrm{fb}^{-1}$ integrated luminosity recorded in 2011.

\section{Summary and outlook}

The performance of the muon system in CMS is excellent: good muon pT resolution, gammar energy resolution and elaborate trigger paths for dimuon or multiple-muon events. The Standard 

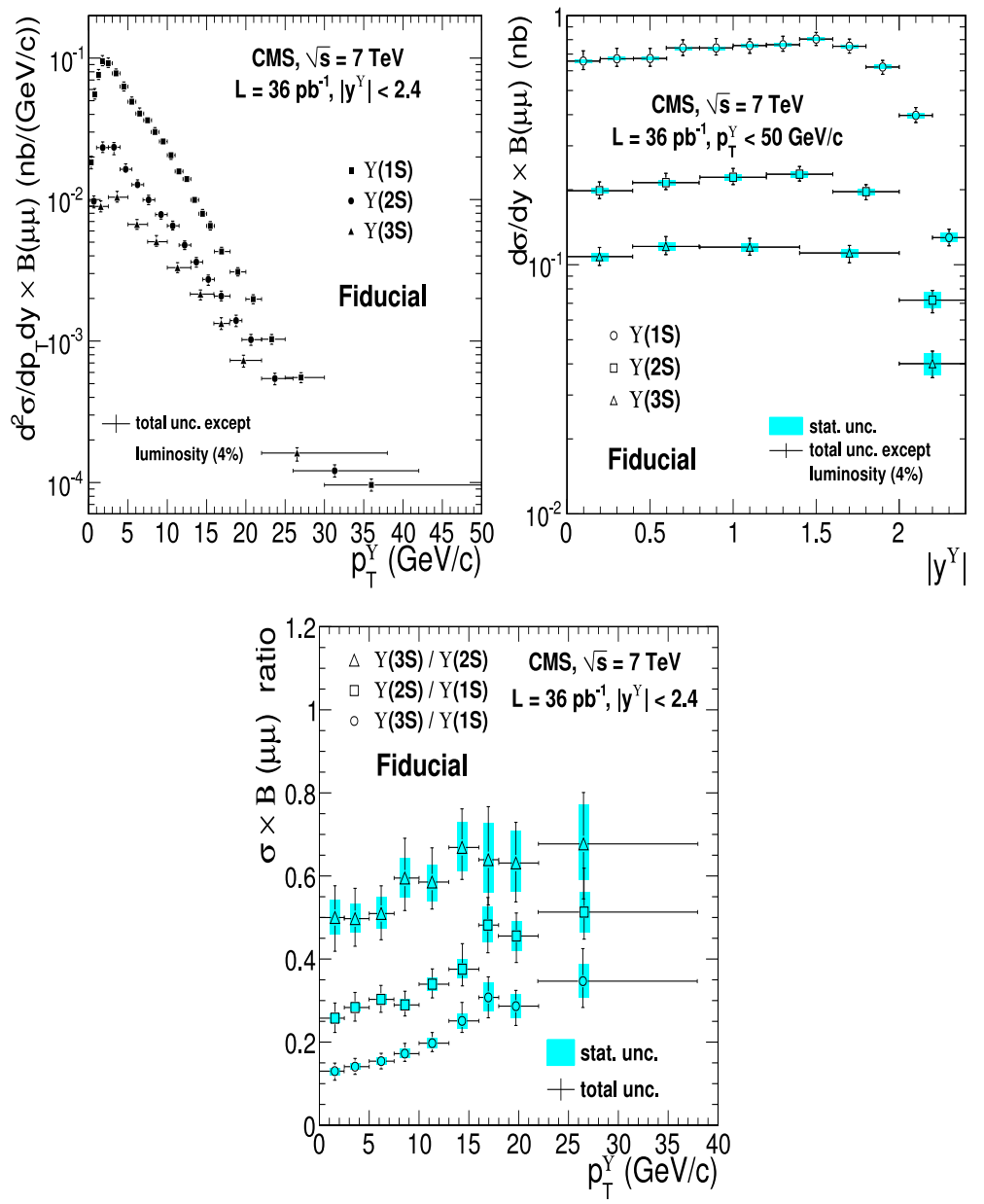

Figure 4: (Left) Differential fiducial cross sections of the $v(n S)$ as a function of $\mathrm{pT}$ in the rapidity range $-2.4<\mathrm{y}<2.4$. The abscissa of the data points is the average $\mathrm{pT}$ in the interval. (Middle) Differential fiducial production cross sections of the $\Upsilon(n S)$ as a function of rapidity. (Right) Ratios of differential fiducial cross sections of the $\Upsilon(n S)$ as a function of $\mathrm{pT}$ in the rapidity range $-2.4<\mathrm{y}<2.4$.

model was tested in quarkonium production and impressive agreement was obtained. The cross sections were measured up to unprecedented $J / \psi$ pT. The cross sections for prompt $J / \psi$ and $\psi(2 S)$ and $\Upsilon(n s)$ were measured with high statistics and are in good agreement with NLO NRQCD prediction at $7 \mathrm{TeV} . \chi_{c}$ was observed through photon conversion. The signal to background ratio was excellent and three states $\chi_{c 0}, \chi_{c 1}$ and $\chi_{c 2}$ were disentangled very well. The exotic state $\mathrm{X}(3872)$ was observed at the new energy region with 2 times statistics as large as CDF. Two structures were confirmed in the $J / \psi \phi$ mass spectrum. On the basis of 2011 data, we are analyzing doublbe $J / \psi$ production through $4 \mu$ final state and Bc production through $J / \psi \pi^{+} \pi^{-}$final state.

\section{References}

[1] S. Chatrchyan et al. [CMS Collaboration], $J / \psi$ and $\psi(2 S)$ production in pp collisions at $\sqrt{s}=7 \mathrm{TeV}$, JHEP 02 (2012) 011. 

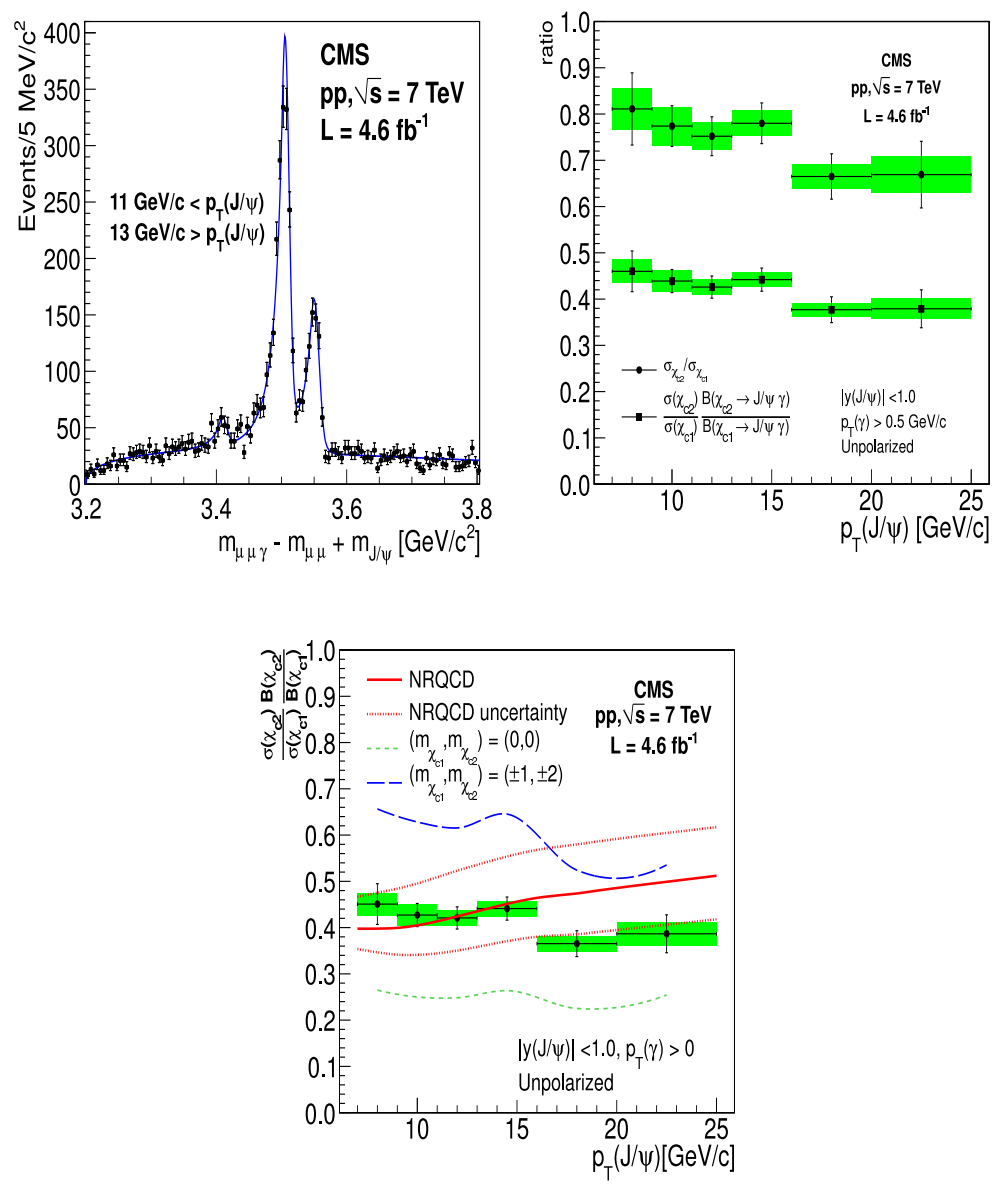

Figure 5: (Left) Mass difference spectrum for selected $\chi_{c 1}$ and $\chi_{c 2}$ candidates. (Middle) Ratio of cross sections and ratio of cross sections multiplied by branching fractions. (Right)Comparison of our measurement with predictions from NRQCD. Since predictions are given in a different kinematic phase space with respect to our measurement, we apply a correction factor derived from Monte Carlo to make the comparison possible. The uncertainty on the correction factors is included as a systematic error in the green area.

[2] Y. Q. MA, K. Wang and K. T. Chao, $J / \psi(\psi(2 S))$ Production at the Tevatron and LHC at $O\left(\alpha^{4} v^{4}\right)$ in Nonrelativistic QCD, Phys. ReV. Lett. 106 (2011) 042002.

[3] CMS Collaboration, Measurement of the $Y(1 S), Y(2 S)$, and $Y(3 S)$ cross sections in pp collisions at $\sqrt{s}$ = $7 \mathrm{TeV}$, arXiv:1303.5900.

[4] CMS Collaboration, Measurement of the relative prompt production rate of $\chi_{c 2}$ and $\chi_{c 1}$ at $\sqrt{s}=7$ TeV, EPJC 72 (2012) 2251.

[5] CMS Collaboration, Measurement of the X(3872) production cross section via decays to J/ $\psi$ pi pi in pp collisions at $\sqrt{s}=7 \mathrm{TeV}$, arXiv:1302.3968.

[6] CMS Collaboration, Observation of structures in the $J / \psi \phi$ spectrum of $B^{+} \rightarrow J / \psi \phi K^{+}$decays, CMS BPH-11-026. 

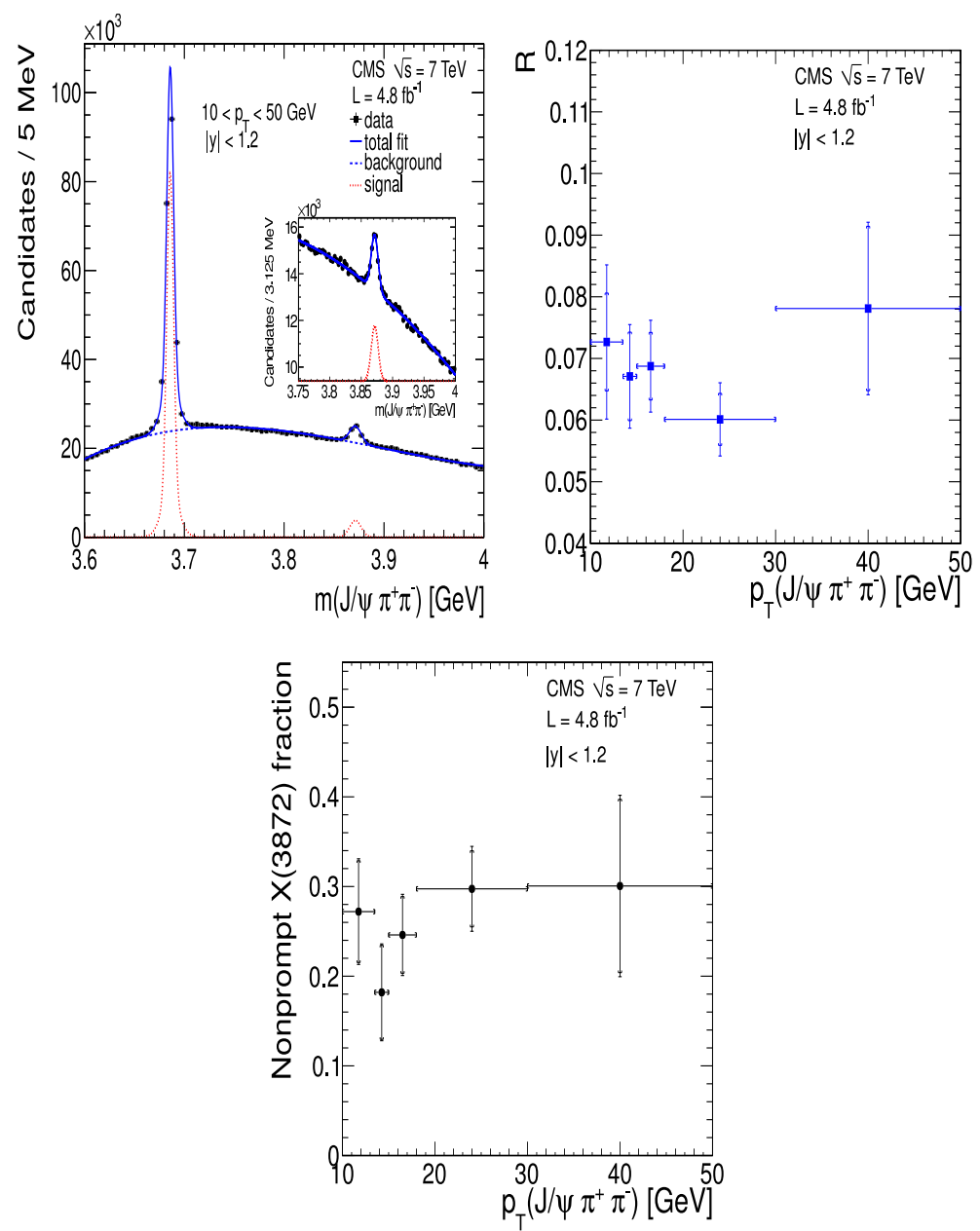

Figure 6: (Left) The $J / \psi \pi+\pi-$ invariant mass spectrum for $10<\mathrm{pT}<50 \mathrm{GeV}$ and $|\mathrm{y}|<1.2$. The lines represent the signal-plus-background fits (solid), the background-only (dashed), and the signal-only (dotted) components. The inset shows an enlargement of the X(3872) mass region. (Middle)Ratio between the $\mathrm{X}(3872)$ and cross sections times branching fractions with acceptance corrections for the muon and pion pairs, as a function of pT. (Right) Measured X(3872) nonprompt fraction, uncorrected for acceptance, as a function of $\mathrm{pT}$. 